\title{
Cortical amyloid beta in cognitively normal elderly adults is associated with decreased network efficiency within the cerebro-cerebellar system
}

\section{Stefanie C. Steininger ${ }^{1}$, Xinyang Liu ${ }^{2}$, Anton Gietl ${ }^{1}$, Michael Wyss ${ }^{3}$, Simon Schreiner ${ }^{1}$, Esmeralda Gruber ${ }^{1}$, Valerie Treyer ${ }^{1,4}$, Andrea Kälin ${ }^{1}$, Sandra Leh ${ }^{1}$, Alfred Buck ${ }^{4}$, Roger M. Nitsch ${ }^{1}$, Klaas P. Prüssmann ${ }^{3}$, Christoph Hock ${ }^{1}$ and Paul G. Unschuld ${ }^{1}$ *}

' Division of Psychiatry Research and Psychogeriatric Medicine, University of Zürich, Zürich, Switzerland

2 Department of Radiology, Brigham and Women's Hospital, Harvard Medical School, Boston, MA, USA

${ }^{3}$ Institute for Biomedical Engineering, University of Zürich, ETH Zürich, Zürich, Switzerland

${ }^{4}$ Division of Nuclear Medicine, University of Zürich, Zürich, Switzerland

\section{Edited by:}

Rodrigo Orlando Kuljiš, Zdrav Mozak Limitada, Chile

Reviewed by:

José M. Delgado-García, University

Pablo de Olavide, Spain

Bogdan O. Popescu, Colentina Clinical

Hospital, Romania

\section{${ }^{*}$ Correspondence:}

Paul G. Unschuld, Division of

Psychiatry Research and

Psychogeriatric Medicine, University

of Zürich, Minervastrasse 145, Zürich

8032, Switzerland

e-mail: paul.unschuld@uzh.ch
Background: Deposition of cortical amyloid beta $(A \beta)$ is a correlate of aging and a risk factor for Alzheimer disease (AD). While several higher order cognitive processes involve functional interactions between cortex and cerebellum, this study aims to investigate effects of cortical $A \beta$ deposition on coupling within the cerebro-cerebellar system.

Methods: We included 15 healthy elderly subjects with normal cognitive performance as assessed by neuropsychological testing. Cortical $A \beta$ was quantified using (11)carbonlabeled Pittsburgh compound B positron-emission-tomography late frame signals. Volumes of brain structures were assessed by applying an automated parcelation algorithm to three dimensional magnetization-prepared rapid gradient-echo T1-weighted images. Basal functional network activity within the cerebro-cerebellar system was assessed using bloodoxygen-level dependent resting state functional magnetic resonance imaging at the high field strength of $7 \mathrm{~T}$ for measuring coupling between cerebellar seeds and cerebral gray matter. A bivariate regression approach was applied for identification of brain regions with significant effects of individual cortical $A \beta$ load on coupling.

Results: Consistent with earlier reports, a significant degree of positive and negative coupling could be observed between cerebellar seeds and cerebral voxels. Significant positive effects of cortical $A \beta$ load on cerebro-cerebellar coupling resulted for cerebral brain regions located in inferior temporal lobe, prefrontal cortex, hippocampus, parahippocampal gyrus, and thalamus.

Conclusion: Our findings indicate that brain amyloidosis in cognitively normal elderly subjects is associated with decreased network efficiency within the cerebro-cerebellar system. While the identified cerebral regions are consistent with established patterns of increased sensitivity for $A \beta$-associated neurodegeneration, additional studies are needed to elucidate the relationship between dysfunction of the cerebro-cerebellar system and risk for AD.

Keywords: cerebellum, fMRI BOLD, functional connectivity, Alzheimer disease, amyloid beta-peptides, aging neuroscience

\section{INTRODUCTION}

Deposition of cortical amyloid beta $(A \beta)$ is a correlate of aging and a risk factor for pathological cognitive decline due to Alzheimer disease (AD) (Sperling et al., 2011; Villemagne et al., 2011, 2013). Cortical $A \beta$ affects various aspects of brain functioning (Morris et al., 2009; Storandt et al., 2009) and may reflect an early stage of AD development (Mormino et al., 2012).

While there is significant variation in elderly persons both regarding the degree of brain amyloidosis (Price et al., 2009; Mormino et al., 2012) as well as cognitive performance levels (Christensen et al., 1999), the term cognitive reserve is used to describe individual resilience against aging-associated decline of brain functionality by maintaining normal cognitive performance despite manifesting cerebral pathology (Stern, 2009). Besides brain amyloidosis, several other processes including tauopathy and inflammation have been suggested to contribute to aging-related disturbances of brain functionality (Gruart et al., 2008; Krstic and Knuesel, 2013) and individual neuronal properties may constitute resilience against cognitive decline in this context (Steffener and Stern, 2012). Functional connectivity at rest has been shown to directly relate to cognitive reserve and has been shown capable of measuring brain amyloidosis-associated functional deficits 
prior to development of cognitive deficits (Hedden et al., 2009; Mormino et al., 2009; Sheline et al., 2010; Oh et al., 2011; Sperling et al., 2011; Sheline and Raichle, 2013).

Functional connectivity is defined by synchronous activity of the blood-oxygen-level dependent (BOLD)-signal at spatially distant loci and indicates coupling between neuronal populations of the brain as a reflection of brain system integrity, such as cognitive processing (Friston et al., 1993; Biswal et al., 1995; van de Ven et al., 2004; Fingelkurts and Kahkonen, 2005; Buckner et al., 2008; Van Dijk et al., 2010). Assessment of functional connectivity has been performed by applying both independent component analysis (ICA) (Calhoun et al., 2001; Beckmann et al., 2005; Greicius et al., 2007; Stevens et al., 2009) and seed-driven approaches (Biswal et al., 1995; Greicius et al., 2003; Fox et al., 2005; Castellanos et al., 2008; Whitfield-Gabrieli et al., 2009; Chai et al., 2012). Seed-driven functional connectivity analysis may delineate disease-related changes in brain systems of interest based on the functional topography (Margulies et al., 2007; Cohen et al., 2008; Di Martino et al., 2008; Roy et al., 2009; Uddin et al., 2010). An integrated approach of assessing seed-based functional connectivity and component-based reduction of noise (Behzadi et al., 2007) allowing second-level random-effect analysis has been published recently (Whitfield-Gabrieli and Nieto-Castanon, 2012).

For the cerebellum, a distinct functional topography has been shown, reflecting connections to distributed circuits within the central nervous system (Stoodley and Schmahmann, 2010; Stoodley et al., 2012). These include loops associated with cognitive processing where the cerebellum exerts a modulating effect that may be impaired in neuropsychiatric disease (Middleton and Strick, 1994; Schmahmann, 1996, 1998; Schmahmann and Caplan, 2006; Schmahmann et al., 2007) and have been shown to functionally interact with intrinsic cerebral network activity (Habas et al., 2009). While coupling within various neural networks and brain systems is known to be affected by AD pathology (Buckner et al., 2008; Sheline et al., 2010; Chhatwal and Sperling, 2012; Matthews et al., 2013), to our knowledge no data has been published so far regarding potential effects of brain amyloidosis on functional integrity of the cerebro-cerebellar system.

Based on these earlier reports, we hypothesized that coupling within the cerebro-cerebellar system would be altered as a function of brain amyloidosis in elderly subjects with normal cognitive performance. Furthermore, brain amyloidosisrelated changes in cerebro-cerebellar coupling may indicate cerebral brain regions with particular vulnerability to $A \beta$-associated pathology.

To answer this question, the current study assessed cortical A $\beta$ load in 15 cognitively normal elderly adults by estimating cortical Pittsburgh compound B (PiB)-retention based on (11)carbonlabeled Pittsburgh compound B positron-emission-tomography (PiB-PET) late frame signals (Mathis et al., 2003; Klunk et al., 2004). In addition, resting state functional magnetic resonance imaging (fMRI) at high field strength of $7 \mathrm{~T}$ was performed to acquire information on regional basal neuronal activity over time at high signal-to-noise ratio (SNR). Cerebral brain regions with significant effects of cortical $A \beta$ load on cerebro-cerebellar coupling were identified by applying second-level random-effect analysis (Whitfield-Gabrieli and Nieto-Castanon, 2012).

\section{MATERIALS AND METHODS STUDY POPULATION}

A total of 15 cognitively healthy elderly subjects without major medical or neuropsychiatric co-morbidity were recruited at the Division of Psychiatry Research and Psychogeriatric Medicine, University of Zürich, Switzerland as approved by the cantonal ethics committees of canton Zürich, Switzerland and Swiss Federal Institute of Technology, respectively (ETH Zürich) in concordance with the declaration of Helsinki (World Medical Association, 1991). Cognitive healthy status of all participants was ascertained by psychiatric examination and neuropsychological testing including mini mental state examination (MMSE) as an initial screen for cognitive impairment (MMSE) (Folstein et al., 1975). In addition, verbal learning and memory test (VLMT) (Helmstaedter and Durwen, 1990; Helmstaedter, 2001) as a modified German version of the auditory VLMT (Lezak, 1983; Müller et al., 1997) was used to assess memory performance (immediate, delayed, and supported recall), Boston Naming Test (BNT) for confrontational word retrieval (Nicholas et al., 1988), memory span [digits forward and backward for short term memory assessment (Gregoire and van der Linden, 1997)], Trail Making Test [ratio of A and B for assessment of mental flexibility (Tombaugh, 2004)] were administered. Body mass index (BMI) was assessed as a general indicator of health and calculated as reported earlier (MacKay, 2010). Exclusion criteria were: cognitive deficits indicating presence of mild cognitive impairment (MCI) or dementia (Petersen et al., 1999; Winblad et al., 2004; Albert et al., 2011), significant medication, or drug abuse that may affect cognition, general magnetic resonance imaging (MRI) exclusion criteria, contraindications against vein puncture, clinically relevant changes in red blood cell count, allergy to the Carbon-11-based PiB positronemission-tomography (PET) tracer or any of its constituents, history of severe allergic reactions to drugs or allergens, serious medical or neuropsychiatric illness, and significant exposure to radiation, respectively.

\section{GENERATION OF (11)CARBON-LABELED PITTSBURGH COMPOUND B TRACER FOR PET}

For cerebral measures of $\mathrm{A} \beta$, (11)carbon-labeled PiB-PET tracer was generated as described earlier (Klunk et al., 2004). In brief, first $11 \mathrm{CO} 2 \mathrm{C}$ was generated using the $14 \mathrm{~N}(p, \alpha) 11 \mathrm{C}$ nuclear reaction on the PET tracer cyclotron $(16.5 \mathrm{MeV}, \mathrm{GE})$ of the PET Center of the Division of Nuclear Medicine, Zürich University Hospital and processed to [11C]-methyl triflate using a AgOTf/C column at $190^{\circ} \mathrm{C}$. By radiolabeling [11C]-methyl triflate with the free amine precursor 6-HO-BTA-0, the PiB-tracer was produced (Solbach et al., 2005) at 99\% radiochemical purity.

\section{PET ACQUISITION FOR ASSESSMENT OF CORTICAL PiB RETENTION}

For the current study, PET scans were performed at the PET Center of the Division of Nuclear Medicine, Zürich University Hospital (GE Discovery RX STE PET/CT scanner). An individual dose equaling $350 \mathrm{MBq}$ of (11)carbon-labeled $\mathrm{PiB}$ was applied using intravenous access to the cubital vein. PET acquisition was dynamic over $70 \mathrm{~min}$, cerebral amyloid deposition was estimated based on late frame signals representing 50-70 min. The emission was corrected for attenuation (CT-based), scatter, 
randoms, and dead time, and reconstructed using a three dimensional (3D) Fourier re-binning (FORE) filtered back projection (FBP) algorithm, resulting in a $128 \times 128 \times 47$ matrix with $2.34 \mathrm{~mm} \times 2.34 \mathrm{~mm} \times 3.27 \mathrm{~mm}$ voxel spacing. As a single measure of individual cortical $\mathrm{A} \beta$ load, cortical $\mathrm{PiB}$ retention scores (cortical PiB) were calculated as reported earlier (Jack et al., 2008; Vandenberghe et al., 2010; Villemagne et al., 2011). In brief, a composite score was calculated using merged cortical PiB-PET intensity values which were referenced to cerebellar intensity after co-registration applying PMOD brain tool (PNEURO) software, Version 3.4 (PMOD Technologies Ltd., Zürich, Switzerland) and transformed to standardized $Z$-scores.

\section{ACQUISITION OF MRI DATA}

Magnetic resonance imaging was performed at the Institute for Biomedical Engineering University of Zurich and ETH Zurich, Switzerland using a Philips 7-T Achieva whole-body scanner (Philips Healthcare, Cleveland, OH, USA) equipped with a nova medical quadrature transmit head coil and 32channel receive coil array (Nova Medical, Wilmington, MA, USA). A high quality T1-weighted 3D magnetization-prepared rapid gradient-echo (MPRAGE) structural brain image covering cerebrum and cerebellum $(\mathrm{TE} / \mathrm{TR}=3.74 / 8.12 \mathrm{~ms}$; resolution: $0.9 \mathrm{~mm} \times 0.9 \mathrm{~mm} \times 0.9 \mathrm{~mm}$; total scan time: $10 \mathrm{~min}$ $54 \mathrm{~s}$; FOV: $220 \mathrm{~mm} \times 160 \mathrm{~mm} \times 200 \mathrm{~mm}$ ) was acquired for volumetric analysis of brain structures and to define cerebellar lobes used as seeds for estimation of cerebro-cerebellar coupling. Echo planar imaging (EPI)-based BOLD fMRI covering cerebrum and cerebellum $(\mathrm{TE} / \mathrm{TR}=25 \mathrm{~ms} / 2500 \mathrm{~ms}$; resolution: $1.72 \mathrm{~mm} \times 1.72 \mathrm{~mm} \times 3 \mathrm{~mm}$; total scan time: $8 \mathrm{~min} 58 \mathrm{~s}$; FOV: $220 \mathrm{~mm} \times 150 \mathrm{~mm} \times 220 \mathrm{~mm}$ ) was used to acquire resting state data, suited for functional connectivity analysis.

\section{POST ACQUISITION IMAGE ANALYSIS AND QUANTIFICATION OF FUNCTIONAL CONNECTIVITY}

Determination of anatomical structures and estimation of volumes was performed by applying the automated imagequantification functions provided by the FreeSurfer image analysis suite (Fischl et al., 2004), using standard operations for construction of cortical models and further data analysis ${ }^{1}$. For improvement of functional SNR and preparation for statistical analysis, fMRI data was preprocessed using MatLab (Version 8.2 for 64-bit processors, MathWorks Inc., Natick, MA, USA) in combination with statistical parametric mapping (SPM, Version 8) ${ }^{2}$ (Friston, 1995) and connectivity toolbox (Conn, Version 13g) ${ }^{3}$ (Whitfield-Gabrieli and Nieto-Castanon, 2012). In brief, individual fMRI volumes were corrected for timing differences between slices, realigned to a reference image (Bspline interpolation based on first image of fMRI data), spatially normalized to Montreal Neurological Institute (MNI) standardized space 4 , corrected for temporal autocorrelation and spatially smoothed using a $6-\mathrm{mm}^{3}$ full-width half-maximum

\footnotetext{
${ }^{1}$ http://surfer.nmr.mgh.harvard.edu/

${ }^{2}$ http://www.fil.ion.ucl.ac.uk/spm/

${ }^{3}$ http://www.nitrc.org/projects/conn/

${ }^{4}$ http://www.mni.mcgill.ca/
}

Gaussian kernel. As described earlier, we used the art-tool $^{5}$ to explore resting state fMRI data for artifacts and to generate a matrix of outlier-time points, which were used as first-level covariates for estimation of cerebro-cerebellar functional connectivity maps (Whitfield-Gabrieli and Nieto-Castanon, 2012). To increase specificity for gray matter signals and to reduce impact of physiological noise such as white matter and cerebrospinal fluid signals, a bandpass filter (0.01$0.1 \mathrm{~Hz}$ ) and the anatomical component-based noise correction method (CompCor) (Behzadi et al., 2007) was applied as described earlier for seed-based functional connectivity analysis (Chai et al., 2011).

Ten bilateral region of interests (ROIs) consistent with reports on regional functional topography (Stoodley et al., 2012) were identified using automated anatomical labeling (AAL) based on the 3D MRI atlas of the human cerebellum (Schmahmann et al., 1999; Tzourio-Mazoyer et al., 2002). Mean BOLD activity within each of these ROIs was estimated and used for definition of 10 "seeds" (Joel et al., 2011): seed \#1 was chosen as a representation of cerebellar lobules I-V, including the region between precentral and primary cerebellar fissure. Seed \#2 includes hemispheric regions between primary and superior posterior cerebellar fissure and represents lobule VI. Seed \#3 represents crus 1 and includes the hemispheric regions between superior posterior and horizontal cerebellar fissures. Seed \#4 represents crus 2 and includes hemispheric regions between horizontal and ansoparamedian fissures. Seed \#5 represents lobule VIIb and includes the region between ansoparamedian and prebiventer cerebellar fissure. Seed \#6 represents lobule VIII and includes the region between prebiventer and secondary fissure. Seed \#7 represents lobule IX and includes the region between secondary and posterolateral fissure. Seed \#8 represents lobule $\mathrm{X}$ and includes cerebellar hemispheres caudal of the posterolateral fissure. The connectivity toolbox (WhitfieldGabrieli and Nieto-Castanon, 2012) was used to first generate functional connectivity maps for each of the 10 cerebellar seeds, representing cerebral voxels with significant BOLD synchrony. Potential effects of cortical $\mathrm{PiB}$ retention (as a measure of cortical A $\beta$ deposition) on cerebro-cerebellar coupling were tested by assessing significant second-level effects on the voxel-level using a bivariate regression approach. Adjustment of significance levels for multiple comparisons were performed as suggested earlier (Chai et al., 2011) by applying a height threshold of $p=0.001$ and false discovery rate (FDR) correction for $\alpha=0.05$ for cluster extent as implemented in the connectivity toolbox software package (Benjamini and Hochberg, 1995; Whitfield-Gabrieli and Nieto-Castanon, 2012). Size of clusters and included brain regions were determined using the $x j V i e w-t o o l b o x^{6}$ and anatomical data as provided by the AAL 3D MRI atlas of the human brain (TzourioMazoyer et al., 2002). MatLab statistical toolbox (Version 8.3, MathWorks Inc., Natick, MA, USA) was used for testing hypotheses on group differences by applying unpaired Student's $t$-test $(p)$ and hypotheses on statistical dependence by applying Pearson correlation analysis $(r, p)$.

\footnotetext{
${ }^{5}$ http://www.nitrc.org/projects/artifact_detect

${ }^{6}$ www.alivelearn.net/xjview8/xjview.m
} 
Table 1 | Demographics of the studied sample including neuropsychological test results.

\begin{tabular}{lccc}
\hline & Mean (SD) & Correlation age \\
\cline { 3 - 4 } & & & \\
& & $\boldsymbol{r}$ & $\boldsymbol{p}$ \\
\hline N (females/males) & $15(7 / 8)$ & - & - \\
Age (years) & $68(5)$ & - & - \\
Education (years) & $14.8(2.11)$ & 0.33 & 0.22 \\
Body mass index (BMI) & $25.63(3.84)$ & 0.18 & 0.53 \\
Cortical PiB retention & $1.24(0.33)$ & -0.42 & 0.12 \\
Mini mental state examination (MMSE) & $29.47(0.92)$ & 0.1 & 0.73 \\
VMLT: immediate recall & $11.40(2.23)$ & 0.05 & 0.86 \\
VMLT: delayed recall & $10.80(2.46)$ & 0.16 & 0.57 \\
VMLT: supported recall & $12.13(1.96)$ & -0.31 & 0.26 \\
Boston Naming Test (BNT) & $14.73(0.59)$ & -0.25 & 0.37 \\
Memory span, digits forward & $7.47(1.06)$ & 0.07 & 0.8 \\
Memory span, digits backward & $6.8(1.61)$ & -0.06 & 0.82 \\
Trail Making Test (ratio TMT-A by TMT-B) & $2.21(0.66)$ & -0.34 & 0.22 \\
& & &
\end{tabular}

Indicated are mean values with standard deviations (SD), as well as Pearson correlation coefficients ( $r$ ) as a measure of relationship with age. $p$-Values indicate a significant relationship at $p<0.05$.

\section{RESULTS}

\section{NEUROPSYCHOLOGICAL TESTING INDICATES NORMAL COGNITIVE PERFORMANCE LEVELS IN THE STUDY POPULATION}

Mean [standard deviation (SD)] values for MMSE were 29.47 (0.92); VLMT (immediate, delayed, and supported recall, respectively) were 11.40 (2.23), 10.80 (2.46), 12.13 (1.96); BNT were 14.73 (0.59); memory span digits forward 7.47 (1.06), digits backward (1.61); and Trail Making Test ratio A by B: $2.21(0.66)$. No significant relationships between age and neuropsychological test performance scores could be observed, as indicated by Pearson correlation analysis (Table 1). Moreover, mean age was 70 years (SD 5 years), mean time of education was 14.8 years (SD 2.11). Mean BMI was 25.63 (SD 3.84), significant medical illness could be excluded based on information gathered during medical history.

\section{HIGHER AGE IS ASSOCIATED WITH INCREASED VOLUMES OF BRAIN STEM AND RIGHT AMYGDALA IN INDIVIDUALS WITH NORMAL COGNITIVE PERFORMANCE}

Sequence-independent segmentation of magnetic resonance images was applied for determination of anatomical structures and estimation of regional volumes as described earlier for cerebral and cerebellar structures (Fischl et al., 2004). Pearson correlation coefficients were calculated as indicators of statistically significant relationship between regional volumes with cortical A $\beta$ (as inferred by cortical $\mathrm{PiB}$ retention) and age, respectively. A relationship between age and volume that was significant when no correction for multiple testing was performed, could be observed for the brain stem $(r=0.53, p=0.04)$ and right amygdala $(r=0.68$, $p=0.01)$. No significant relationships could be observed between $\mathrm{PiB}$ retention and volumes in our study population of elderly individuals with normal cognitive performance (Table 2).

\section{BOLD SYNCHRONY BETWEEN CEREBELLAR SEEDS AND CEREBRAL GRAY MATTER INDICATES SIGNIFICANT CEREBRO-CEREBELLAR COUPLING}

Based on anatomical data provided by the 3D MRI atlas of the human cerebellum (Schmahmann et al., 1999; Tzourio-Mazoyer et al., 2002), eight ROIs were generated. The ROI used for seed \#1 representing cerebellar lobules I-V included a volume of 22,406 voxels; seed \#2 representing lobule VI included 23,988 voxels; seed \#3 representing crus 1 included 35,584 voxels; seed \#4 representing crus 2 included 25,747 voxels; seed \#5 representing lobule VIIb included 13,236 voxels; seed \#6 representing lobule VIII included 24,420 voxels; seed \#7 representing lobule IX included 9538 voxels; Seed \#8 representing lobule X included 1835 voxels (Figure 1). The connectivity toolbox (Whitfield-Gabrieli and Nieto-Castanon, 2012) was used to identify the extent of BOLD synchrony between each of the eight cerebellar ROIs and cerebral voxels applying a seed to voxel approach. At a height threshold of $p=0.001$ and cluster extent threshold of $p=0.05$ (FDR-corrected), significant coupling between cerebellar seeds and cerebral cortical and subcortical structures could be observed as indicated by the AAL-atlas (Tzourio-Mazoyer et al., 2002) (Figure 2): for seed \#1 (representing cerebellar lobules I-V) significant positive BOLD synchrony could be observed for a cerebral brain area of 47,891 voxels including occipital, temporal, limbic, and parietal lobes. Significant negative BOLD synchrony could be observed for 37,563 voxels located in the brain stem, pons, medulla, and fusiform gyrus. For seed \#2 (representing lobule VI), significant positive BOLD synchrony could be observed for 46,349 voxels in the temporal, occipital, and parietal lobes. Significant negative BOLD synchrony could be observed for 45,619 voxels in the limbic lobe, uncus, medulla, and brain stem. For seed \#3 (representing crus 1), significant positive BOLD synchrony could be observed for 45,554 voxels in the temporal, occipital, and parietal lobes. Significant negative BOLD synchrony could be observed for 43,099 voxels in the limbic, lobe, uncus, parahippocampal gyrus, and frontal lobes. For seed \#4 (representing crus 2), significant positive BOLD synchrony could be observed for 34,463 voxels including the temporal, occipital, frontal lobes, and middle temporal gyrus, respectively. Significant negative BOLD synchrony could be observed for 34,452 voxels in the brain regions temporal lobe, Brodmann area 20 , and parietal and limbic lobes. For seed \#5 (representing lobule VIIb), significant positive BOLD synchrony could be observed for 32,646 voxels located in temporal, occipital frontal lobes, and pons. Significant negative BOLD synchrony could be observed for 32,943 voxels including frontal, limbic lobes, sub-lobar regions, and the parahippocampal gyrus. For seed \#6 (representing lobule VIII), significant positive BOLD synchrony could be observed for 37,445 voxels including the brain regions' temporal lobe, superior frontal gyrus, Brodmann area 9, and Brodmann area 10. Significant negative BOLD synchrony could be observed for 37,382 voxels in the limbic lobe, parahippocampal gyrus, putamen, and temporal lobe. For seed \#7 (representing lobule IX), significant positive BOLD synchrony could be observed for 27,462 voxels in the temporal, occipital, and limbic lobes as well as parahippocampal gyrus. Significant negative BOLD synchrony could be observed for 26,263 voxels in frontal lobe, putamen, Brodmann area 11, and Brodmann area 47 . For seed \#8 (representing lobule X), significant 
Table 2 | Volumes of brain structures as derived from the 7-TT1 MPRAGE images.

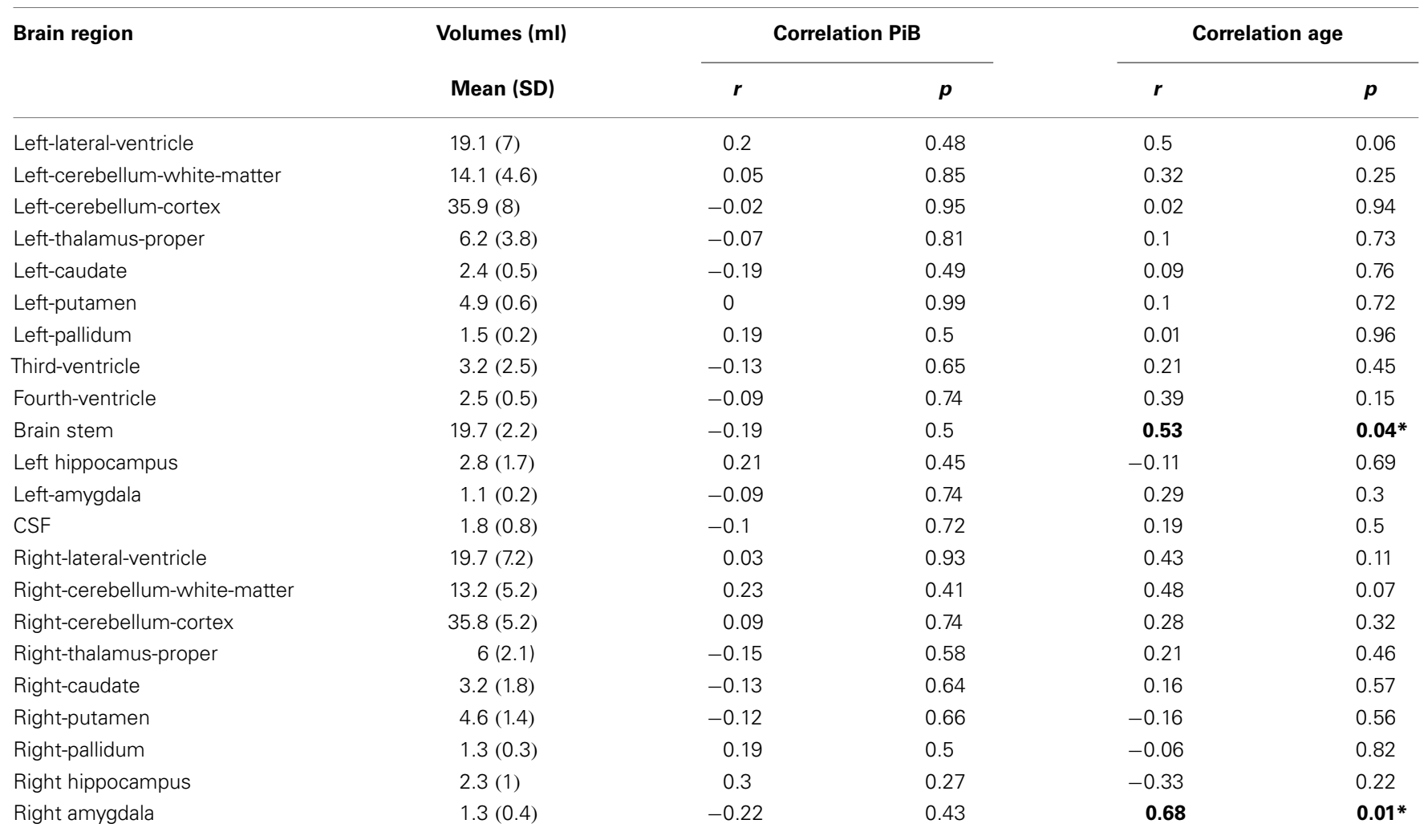

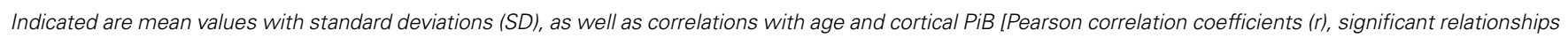
at $p<0.05$ are indicated by "*"]. Bold font indicates significant relationships.

positive BOLD synchrony could be observed for 22,907 voxels in the temporal lobe, pons, brain stem, and limbic lobe. Significant negative BOLD synchrony could be observed for 22,348 voxels in the frontal lobe, Brodmann area 11, limbic lobe, and anterior cingulate. Mean (SD) voxel size of clusters with positive BOLD synchrony was 36,840 (9213) and for negative BOLD synchrony 34,959 (7852). There was no significant difference in size between clusters of positive and negative BOLD synchrony $(p=0.67)$.

\section{CORTICAL A $\beta$ IS ASSOCIATED WITH IMPAIRED EFFICIENCY OF CEREBRO-CEREBELLAR COUPLING}

At a height threshold of $p=0.001$ and cluster extent threshold of $p=0.05$ (FDR-corrected), cerebral voxels with significant positive second-level effects of cortical A $\beta$ (as estimated by cortical PiB retention) on coupling with cerebellar seeds could be observed for seed \#2 (lobule VI) and seed \#8 (lobule X), indicating increased BOLD synchrony as a correlate of cortical $A \beta$. No cerebral voxels with significant negative effects of cortical $A \beta$ could be observed. Clusters with significant cerebral voxels were extracted using algorithms provided by the connectivity tool box (Whitfield-Gabrieli and Nieto-Castanon, 2012) and anatomically categorized based on information provided by the AAL-atlas (Tzourio-Mazoyer et al., 2002). Effects of cortical A $\beta$ (as estimated by cortical PiB retention) on coupling with seed \#2 (lobule VI) were observable for a unilateral cluster of 426 voxels with a peak at MNI: 52, -38 , -24 . Brain regions included, as indicated by the AAL MRI atlas
(Tzourio-Mazoyer et al., 2002), were primarily located in inferior and lateral surfaces of the right temporal lobe (359 voxels), including fusiform gyrus (178 voxels), inferior temporal gyrus (126 voxels), middle temporal gyrus (53 voxels). Effects of cortical A $\beta$ on coupling with seed \#8 (lobule $\mathrm{X}$ ) were observable for a bilateral cluster including 2205 voxels with a peak at MNI: 24, -12, -28 . This cluster included voxels primarily located in the limbic (535 voxels) and temporal lobes (487 voxels). As indicated by the AAL MRI atlas, voxels were located in the following brain regions: right hippocampus (335 voxels), right parahippocampal gyrus (290 voxels), right thalamus (244 voxels), left thalamus (244 voxels), midbrain (240 voxels), left parahippocampal gyrus (173 voxels), right brain stem (148 voxels), left brain stem (91 voxels), left hippocampus (89 voxels) (Figure 3). No cerebral voxels with significant effects of age on BOLD synchrony could be detected in our sample when applying a height threshold of $p=0.001$ and FDR-corrected cluster extent threshold of $p=0.05$.

\section{DISCUSSION}

By performing resting state $\mathrm{AMRI}$ at high field strength of $7 \mathrm{~T}$ and assessment of cortical $\mathrm{A} \beta$ by PiB-PET in a sample of cognitively normal elderly subjects, a significant effect of cortical $A \beta$ on cerebro-cerebellar coupling was observable. Our data indicate decreased network efficiency that particularly affects brain regions located in the limbic lobe, temporal lobe, and subcortical gray nuclei including hippocampus, parahippocampal gyrus, 


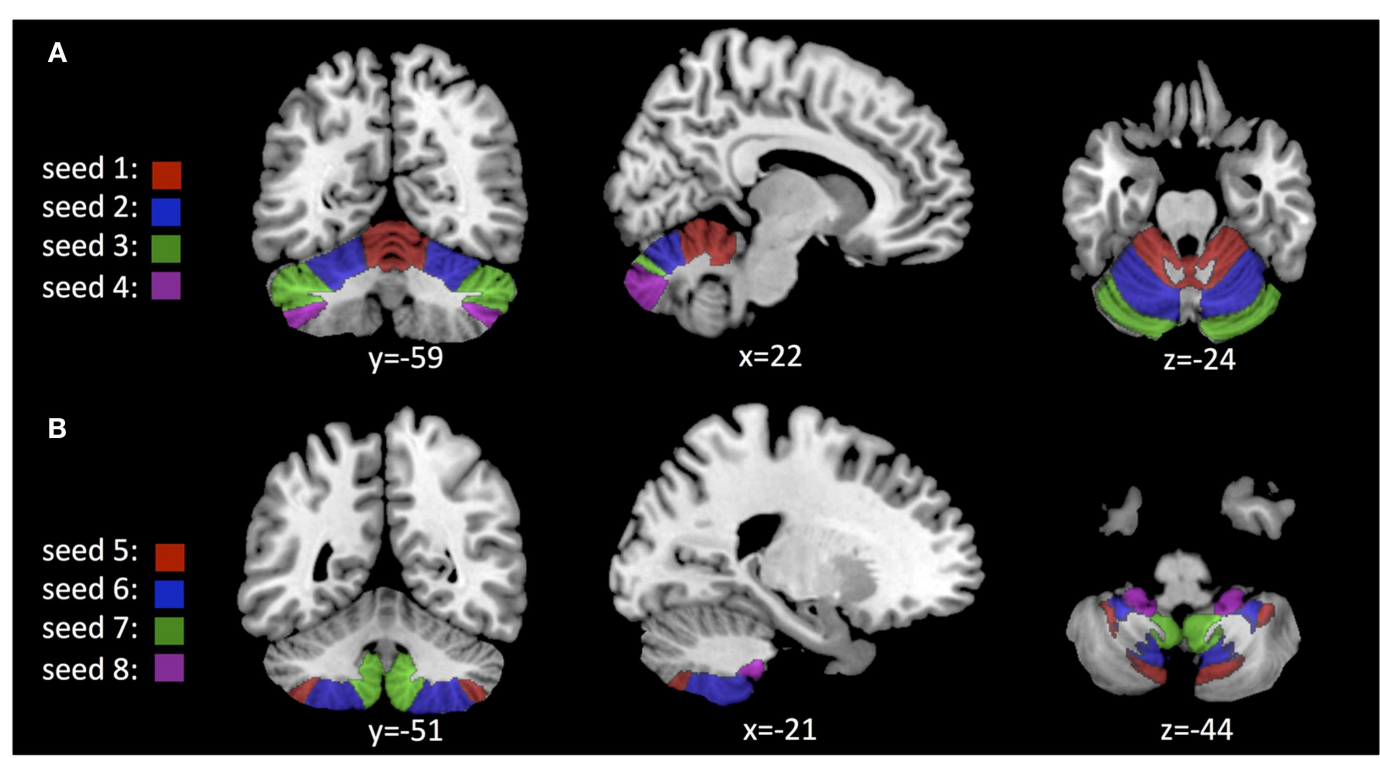

FIGURE 1 | Location of seeds used for analysis of cerebro-cerebellar coupling as derived from the AAL 3D MRI atlas (Schmahmann et al., 1999; Tzourio-Mazoyer et al., 2002): (A) Seeds \#1-4, representing cerebellar lobules I-V; VI; crus 1; crus 2. (B) Seeds \#5-8, representing lobules VIIb; VIII; IX; X.
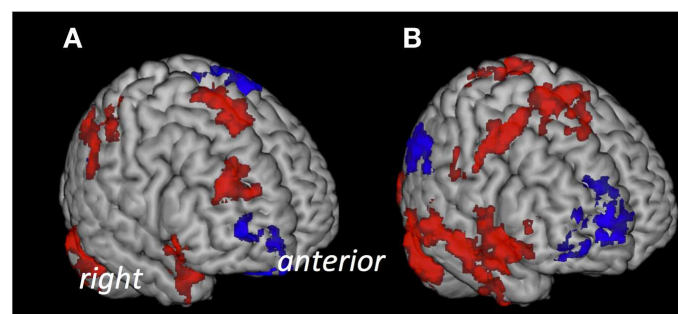

$\mathbf{E}$
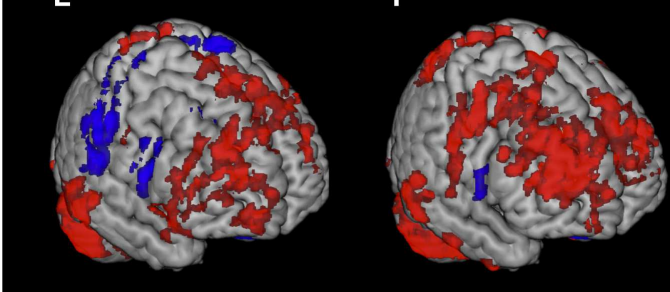

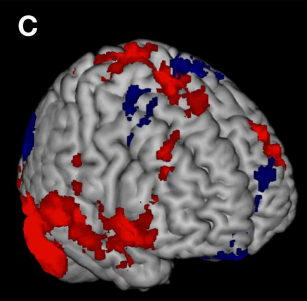

G

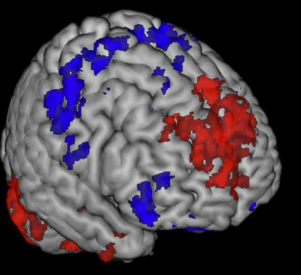

D

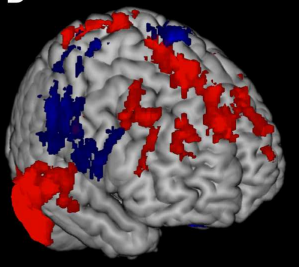

H

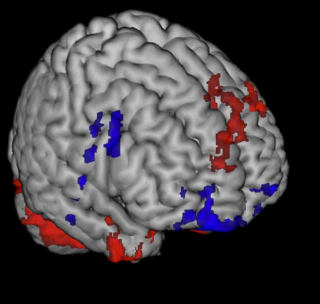

FIGURE 2 | Cerebral voxels with significant coupling to the respective seeds at rest. Areas with "positive" connectivity are indicated in red,

"negative" connectivity in blue: (A) Seed \#1 (cerebellar lobules I-V),
(B) seed \#2 (lobule VI), (C) seed \#3 (crus 1), (D) seed \#4 (crus 2), (E) seed \#5 (lobule VIIb), (F) seed \#6 (lobule VIII), (G) seed \#7 (lobule IX), (H) seed \#8 (lobule X). fusiform gyrus, inferior temporal gyrus, and thalamus. No effect of cortical $A \beta$ on volumes of brain regions was observable, which appears consistent with the high level of cognitive performance measured in the study sample and also earlier considerations that functional alterations may precede brain atrophy in $\mathrm{A} \beta$-associated neurodegenerative illness such as AD.

All participants included in the current study displayed normal cognitive performance levels, as indicated by neuropsychological testing including MMSE (Folstein et al., 1975),
VLMT (Helmstaedter and Durwen, 1990), BNT (Nicholas et al., 1988), memory span (Gregoire and van der Linden, 1997), and mental flexibility (Tombaugh, 2004). In our sample, no relationship between lower test performance and higher age was observable, indicating absence of aging-related cognitive decline in the studied sample.

Pittsburgh compound B positron-emission-tomography (Mathis et al., 2003; Klunk et al., 2004) is the most extensively studied and best validated tracer for quantification of $A \beta$ accumulation 

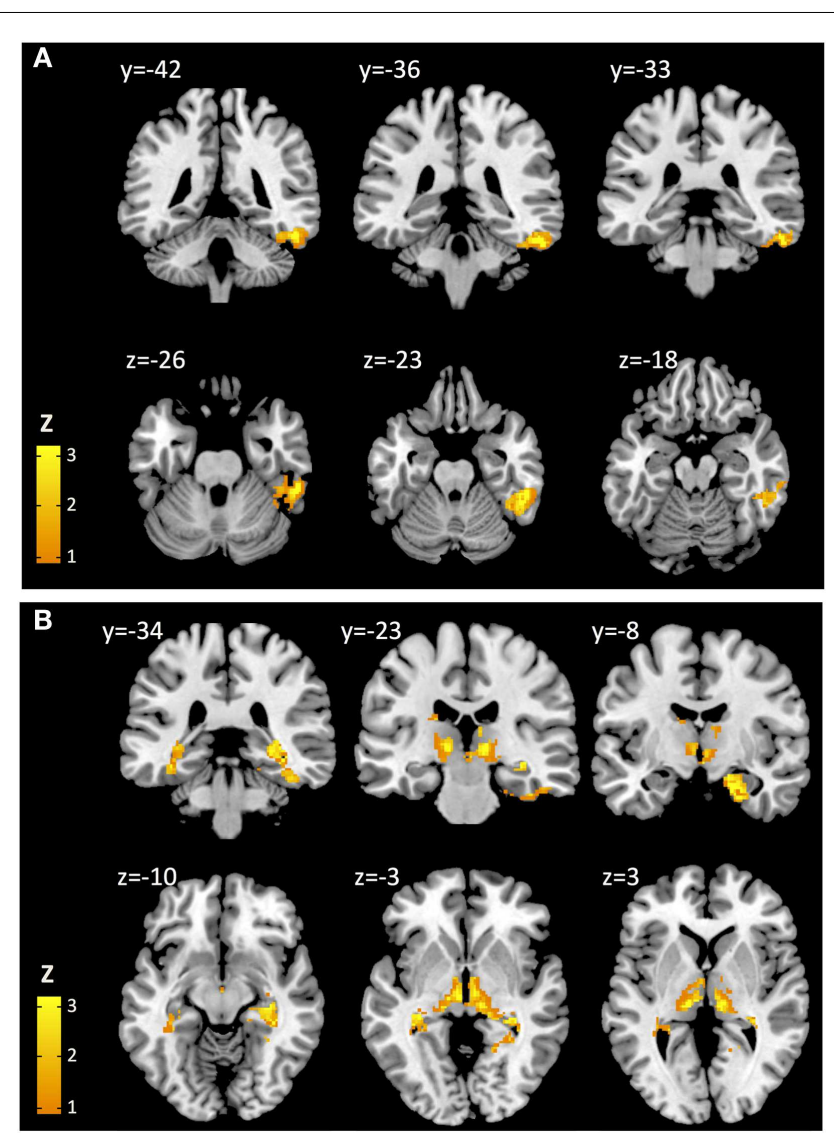

FIGURE 3 | Cerebral voxels (MNI) with significant positive effects of cortical $A \beta$ on cerebro-cerebellar coupling for seeds representing cerebellar lobe $\mathrm{VI}(\mathrm{A})$ and lobe $\mathrm{X}(\mathrm{B})$, respectively.

in the human brain (Rabinovici and Jagust, 2009) and was used in the current study to assess the degree of individual cerebral $A \beta$ load in all study participants. While cerebral $A \beta$ deposition is a hallmark of AD (Alzheimer, 1907; Hock and Nitsch, 2000; Albert et al., 2011), recent studies applying PiB-PET have demonstrated an association of individual $A \beta$ load with increased risk for agingrelated cognitive decline and incidence of $\mathrm{AD}$, respectively (Jagust et al., 2009; Sperling et al., 2011; Shaffer et al., 2013; Wirth et al., 2013). To obtain a single measure of individual cortical $A \beta$ load, we calculated cortical PiB retention scores (cortical PiB), indicating global cortical uptake of the PiB-PET tracer normalized to intensity of the cerebellum (Jack et al., 2008; Vandenberghe et al., 2010; Villemagne et al., 2011).

Processing of cognitive tasks has been shown to be associated with distinct neuronal systems whose activity is reflected by several functional networks of spatially distant brain regions, linked by synchronous BOLD activity both under challenge as well as at rest (Biswal et al., 1995; Woodward et al., 2006; Buckner et al., 2008; Fair et al., 2009). While integrity of functional brain networks has been shown to be impaired in the context of many neuropsychiatric illnesses (Calhoun et al., 2008; Buckner et al., 2009; Whitfield-Gabrieli and Ford, 2012; Unschuld et al., 2013a,b), there is a concatenation of studies that indicate cerebral
$A \beta$ accumulation as a significant interference factor on physiological brain network activity (Woodward et al., 2006; Hedden et al., 2009; Sheline et al., 2010; Oh et al., 2011). However, while there are publications reporting effects of $A \beta$ on integrity of many brain networks, to our knowledge there are no studies published that investigate effects of $A \beta$ on cerebro-cerebellar coupling.

For this study, resting state fMRI at high field strength of $7 \mathrm{~T}$ was used for assessment of basal neural activity at increased SNRs, which has been demonstrated to provide increased sensitivity for BOLD activity and assessment of intrinsic network activity compared to lower field strength MRI (Donahue et al., 2010, 2011; Lenglet et al., 2012; Theysohn et al., 2013; Zhan et al., 2013). To measure functional connectivity within the cerebro-cerebellar system and its potential alteration in a context of cortical $A \beta$ load, as indicated by cortical $\mathrm{PiB}$ retention scores, a bivariate regression analysis was applied using algorithms provided by the connectivity toolbox (Whitfield-Gabrieli and Nieto-Castanon, 2012). While earlier studies investigating functional connectivity in the cerebrocerebellar system used both ICA (Habas et al., 2009) as well as seed-based approaches (He et al., 2004; Allen et al., 2005), we chose a seed-based approach as it allows specific investigation of functional brain systems and produces results that are consistent with ICA experiments (Joel et al., 2011). Based on the AAL 3D MRI atlas (Schmahmann et al., 1999; Tzourio-Mazoyer et al., 2002) and earlier information on functional topography of the cerebellum (Stoodley et al., 2010, 2012), eight cerebellar ROI were chosen as seeds. As brain atrophy is a frequent finding associated with aging (Scahill et al., 2003) and also has been shown to relate to A $\beta$ load in AD (de Leon et al., 1997; de Leon and Klunk, 2006; Storandt et al., 2009), volumes of cortical and subcortical brain regions were assessed by applying the FreeSurfer analysis suite (Fischl et al., 2002, 2004) and then tested for relationship with cortical $A \beta$. To be able to distinguish effects due to cortical $A \beta$ from effects attributable to other factors associated with aging, also relationship between cerebro-cerebellar coupling and brain volumes, respectively, with age was tested.

Our data are consistent with earlier reports as a significant degree of functional connectivity could be observed between cerebellar seeds and cerebral voxels, many of whom are attributable to well-established functional networks (Allen et al., 2005; Habas et al., 2009). However, connectivity maps in the current dataset involve structures that diverge from earlier assumed roles for the respective cerebellar subregions (Stoodley and Schmahmann, 2009; Stoodley et al., 2012). This may be explained by the fact that earlier studies have applied task-paradigms for mapping cerebellar cognitive processing, whereas we assessed basal neural activity at rest. In addition, as our data has been acquired at high magnetic field strength of $7 \mathrm{~T}$, the fact that additional network activity may be detected due to increased SNRs needs to be considered when comparing network properties to studies performed at lower field strength as discussed earlier (Zhan et al., 2013). Moreover, our data indicate a significant effect of cortical $\mathrm{PiB}$ on cerebro-cerebellar coupling that is observable for seeds representing lobule VI and $\mathrm{X}$. While lobule VI has been shown earlier to be involved in executive function as well as salience processing (Allen et al., 2005; Stoodley and Schmahmann, 2009), to our knowledge so far no fMRI studies inferring a functional role for cerebellar lobule X 
have been published. However, the absence of motor syndrome after ischemic infarction of the flocculonodular lobe may indicate relevance of lobule $\mathrm{X}$ for non-motor tasks and cognitive processing (Schmahmann et al., 2009). We could identify cerebral voxels with an increase of coupling associated with higher cortical PiB in brain regions including hippocampus, parahippocampal gyrus, fusiform gyrus, and inferior temporal gyrus but also thalamus. While all of these brain regions have been shown to be affected by atrophy in a context of $\mathrm{AD}$ and brain $\mathrm{A} \beta$ deposition (Bakker et al., 2008; Jack et al., 2008; Storandt et al., 2009; Ballard et al., 2011), our finding of increased connectivity might be a reflection of $\mathrm{AD}$ pathology-associated brain change, resulting in reduced network efficiency. Moreover, the here described effects of cortical A $\beta$ may indicate increased vulnerability of the cerebro-cerebellar system, consistent with earlier considerations on neuronal changes in presymptomatic AD (Mormino et al., 2009; Jagust, 2013). As tasks carried out by the cerebro-cerebellar system include cognitive processes such as visual-spatial, executive function, and workingmemory (Schmahmann and Pandya, 2008; Stoodley, 2012), which frequently are affected in subjects diagnosed with MCI or AD (Hock and Nitsch, 2000; Storandt et al., 2009; Albert et al., 2011; Ballard et al., 2011), prospective longitudinal studies may reveal the impact of cerebro-cerebellar dysfunction on cognitive dysfunction in $\mathrm{AD}$. The fact that we find effects of $\mathrm{A} \beta$ on cerebro-cerebellar connectivity but no relationship between $A \beta$ and volumes may be consistent with earlier reports on functional changes potentially preceding brain atrophy in presymptomatic AD (Sperling et al., 2009; Sheline et al., 2010; Sheline and Raichle, 2013). However, we do find a nominally significant association of higher brain stem volume and amygdala volume with age, which may reflect resilience for maintaining a normal level of cognitive functioning at higher age and potentially interacts with other neuronal factors of cognitive reserve (Stern, 2009; Steffener and Stern, 2012). As $p$-values indicating significance of this relationship were not corrected for multiple comparisons, this finding on potential relevance of brain stem and amygdala volume needs to be treated with caution. While we do not find an effect of aging on cerebrocerebellar coupling, our data does not provide evidence against the possibility that there nevertheless may exist a relationship between higher age and functionality of the cerebro-cerebellar system that might be observable in a larger sample that represents a wider age range of study participants. Moreover, recent data derived both from animal and neuropathological studies indicates that several pathological processes may take place in parallel to or as an upstream event of brain $\mathrm{A} \beta$ accumulation (Gruart et al., 2008; Krstic and Knuesel, 2013). While the experimental setup of our study only allowed to assess effects of $A \beta$ load on cortico-cerebellar coupling, potential modulating effects reflecting, e.g., tauopathy or regional sublevel inflammation thus could not be taken into account.

Taken together, by using in vivo PiB-PET imaging, we could demonstrate that high levels of cortical $A \beta$ are associated with aberrant cortico-cerebellar coupling in cognitively normal elderly individuals. Cerebral regions particularly affected by altered coupling are consistent with established patterns of increased sensitivity for pathological brain change in AD. These findings suggest that $A \beta$ is linked to functional reorganization within the cerebro-cerebellar system, taking place in elderly individuals with normal cognitive performance. Additional longitudinal studies including patients with $\mathrm{MCI}$ and $\mathrm{AD}$ are necessary to figure out up to what degree $A \beta$-associated alterations in the cerebro-cerebellar system contribute to clinical symptoms or potentially represent an early stage of $\mathrm{AD}$ with increased risk for progression to dementia.

\section{ACKNOWLEDGMENTS}

We thank all subjects for their study participation. We thank Linjing $\mathrm{Mu}, \mathrm{Ph} . \mathrm{D}$. and Geoff Warnock, Ph.D. from the Division of Nuclear Medicine, University of Zürich, Switzerland for their help in generation of (11) carbon-labeled Pittsburgh Compound-B tracer for Positron-Emission-Tomography (Linjing $\mathrm{Mu}$ ) and calculation of the cortical PiB retention scores (Geoff Warnock). We thank Daniel Summermatter, Ph.D. from Division of Psychiatry Research and Psychogeriatric Medicine, University of Zürich, Switzerland, for help in interpretation of neuropsychological test results. This work was funded by the Swiss National Science Foundation (Schweizerischer Nationalfonds, SNF), the KFSP "Molecular Imaging", institutional support from the Division of Psychiatry Research and Psychogeriatric Medicine, University of Zürich and Institute for Biomedical Engineering, University of Zürich and ETH Zürich, Switzerland.

\section{REFERENCES}

Albert, M. S., Dekosky, S. T., Dickson, D., Dubois, B., Feldman, H. H., Fox, N. C., et al. (2011). The diagnosis of mild cognitive impairment due to Alzheimer's disease: recommendations from the National Institute on Aging-Alzheimer's Association workgroups on diagnostic guidelines for Alzheimer's disease. Alzheimers Dement. 7, 270-279. doi:10.1016/j.jalz.2011.03.008

Allen, G., McColl, R., Barnard, H., Ringe, W. K., Fleckenstein, J., and Cullum, C. M. (2005). Magnetic resonance imaging of cerebellar-prefrontal and cerebellar-parietal functional connectivity. Neuroimage 28, 39-48. doi:10.1016/ j.neuroimage.2005.06.013

Alzheimer, A. (1907). Über eine eigenartige Erkrankung der Hirnrinde. Allgemeine Zeitschrift für Psychiatrie 64, 146-148.

Bakker, A., Kirwan, C. B., Miller, M., and Stark, C. E. (2008). Pattern separation in the human hippocampal CA3 and dentate gyrus. Science 319, 1640-1642. doi:10.1126/science. 1152882

Ballard, C., Gauthier, S., Corbett, A., Brayne, C., Aarsland, D., and Jones, E. (2011). Alzheimer's disease. Lancet 377, 1019-1031. doi:10.1016/S01406736(10)61349-9

Beckmann, C. F., Deluca, M., Devlin, J. T., and Smith, S. M. (2005). Investigations into resting-state connectivity using independent component analysis. Philos. Trans. R. Soc. Lond. B Biol. Sci. 360, 1001-1013. doi:10.1098/rstb.2005.1634

Behzadi, Y., Restom, K., Liau, J., and Liu, T. T. (2007). A component based noise correction method (CompCor) for BOLD and perfusion based fMRI. Neuroimage 37, 90-101. doi:10.1016/j.neuroimage.2007.04.042

Benjamini, Y., and Hochberg, Y. (1995). Controlling the false discovery rate: a practical and powerful approach to multiple testing. J. R. Stat. Soc. Series B Stat. Methodol. 57, 289-300.

Biswal, B., Yetkin, F. Z., Haughton, V. M., and Hyde, J. S. (1995). Functional connectivity in the motor cortex of resting human brain using echo-planar MRI. Magn. Reson. Med. 34, 537-541. doi:10.1002/mrm.1910340409

Buckner, R. L., Andrews-Hanna, J. R., and Schacter, D. L. (2008). The brain's default network: anatomy, function, and relevance to disease. Ann. N. Y. Acad. Sci. 1124, 1-38. doi:10.1196/annals.1440.011

Buckner, R. L., Sepulcre, J., Talukdar, T., Krienen, F. M., Liu, H., Hedden, T., et al. (2009). Cortical hubs revealed by intrinsic functional connectivity: mapping, assessment of stability, and relation to Alzheimer's disease. J. Neurosci. 29, 1860-1873. doi:10.1523/JNEUROSCI.5062-08.2009

Calhoun, V. D., Adali, T., Pearlson, G. D., and Pekar, J. J. (2001). A method for making group inferences from functional MRI data using independent component analysis. Hum. Brain Mapp. 14, 140-151. doi:10.1002/hbm.1048 
Calhoun, V. D., Maciejewski, P. K., Pearlson, G. D., and Kiehl, K. A. (2008). Temporal lobe and "default" hemodynamic brain modes discriminate between schizophrenia and bipolar disorder. Hum. Brain Mapp. 29, 1265-1275. doi:10.1002/hbm. 20463

Castellanos, F. X., Margulies, D. S., Kelly, C., Uddin, L. Q., Ghaffari, M., Kirsch, A., et al. (2008). Cingulate-precuneus interactions: a new locus of dysfunction in adult attention-deficit/hyperactivity disorder. Biol. Psychiatry 63, 332-337. doi:10.1016/j.biopsych.2007.06.025

Chai, X. J., Castanon, A. N., Ongur, D., and Whitfield-Gabrieli, S. (2012). Anticorrelations in resting state networks without global signal regression. Neuroimage 59, 1420-1428. doi:10.1016/j.neuroimage.2011.08.048

Chai, X. J., Whitfield-Gabrieli, S., Shinn, A. K., Gabrieli, J. D., Nieto-Castanon, A., McCarthy, J. M., et al. (2011). Abnormal medial prefrontal cortex resting-state connectivity in bipolar disorder and schizophrenia. Neuropsychopharmacology 36, 2009-2017. doi:10.1038/npp.2011.88

Chhatwal, J. P., and Sperling, R. A. (2012). Functional MRI of mnemonic networks across the spectrum of normal aging, mild cognitive impairment, and Alzheimer's disease. J. Alzheimers Dis. 31(Suppl. 3), S155-S167. doi:10.3233/ JAD-2012-120730

Christensen, H., MacKinnon, A. J., Korten, A. E., Jorm, A. F., Henderson, A. S., Jacomb, P., et al. (1999). An analysis of diversity in the cognitive performance of elderly community dwellers: individual differences in change scores as a function of age. Psychol. Aging 14, 365-379. doi:10.1037/0882-7974.14.3.365

Cohen, A. L., Fair, D. A., Dosenbach, N. U., Miezin, F. M., Dierker, D., Van Essen, D. C., et al. (2008). Defining functional areas in individual human brains using resting functional connectivity MRI. Neuroimage 41, 45-57. doi:10.1016/j.neuroimage. 2008.01.066

de Leon, M. J., Convit, A., Desanti, S., Bobinski, M., George, A. E., Wisniewski, H. M., et al. (1997). Contribution of structural neuroimaging to the early diagnosis of Alzheimer's disease. Int. Psychogeriatr. 9(Suppl. 1), 183-190. doi:10.1017/ S1041610297004900

de Leon, M. J., and Klunk, W. (2006). Biomarkers for the early diagnosis of Alzheimer's disease. Lancet Neurol. 5, 198-199. doi:10.1016/S1474-4422(06) 70357-X

Di Martino, A., Scheres, A., Margulies, D. S., Kelly, A. M., Uddin, L. Q., Shehzad, Z., et al. (2008). Functional connectivity of human striatum: a resting state FMRI study. Cereb. Cortex 18, 2735-2747. doi:10.1093/cercor/bhn041

Donahue, M. J., Hoogduin, H., Smith, S. M., Siero, J. C., Chappell, M., Petridou, N., et al. (2011). Spontaneous blood oxygenation level-dependent fMRI signal is modulated by behavioral state and correlates with evoked response in sensorimotor cortex: a 7.0-T fMRI study. Hum. Brain Mapp. 33, 511-522. doi:10.1002/hbm. 21228

Donahue, M. J., Hoogduin, H., Van Zijl, P., Jezzard, P., Luijten, P. R., and Hendrikse, J. (2010). Blood oxygenation level-dependent (BOLD) total and extravascular signal changes and $\mathrm{dR} 2^{*}$ in human visual cortex at $1.5,3.0$ and 7.0 T. NMR. Biomed. 23, 1-11. doi:10.1002/nbm.1552

Fair, D. A., Cohen, A. L., Power, J. D., Dosenbach, N. U., Church, J. A., Miezin, F. M., et al. (2009). Functional brain networks develop from a "local to distributed" organization. PLoS Comput. Biol. 5:e1000381. doi:10.1371/journal.pcbi.1000381

Fingelkurts, A. A., and Kahkonen, S. (2005). Functional connectivity in the brain is it an elusive concept? Neurosci. Biobehav. Rev. 28, 827-836. doi:10.1016/j. neubiorev.2004.10.009

Fischl, B., Salat, D. H., Busa, E., Albert, M., Dieterich, M., Haselgrove, C., et al. (2002). Whole brain segmentation: automated labeling of neuroanatomical structures in the human brain. Neuron 33, 341-355. doi:10.1016/S0896-6273(02)00569-X

Fischl, B., Salat, D. H., Van Der Kouwe, A. J., Makris, N., Segonne, F., Quinn, B. T., et al. (2004). Sequence-independent segmentation of magnetic resonance images. Neuroimage 23(Suppl. 1), S69-S84. doi:10.1016/j.neuroimage.2004.07.016

Folstein, M. F., Folstein, S. E., and McHugh, P. R. (1975). "Mini-mental state". A practical method for grading the cognitive state of patients for the clinician. J. Psychiatr. Res. 12, 189-198. doi:10.1016/0022-3956(75)90026-6

Fox, M. D., Snyder, A. Z., Vincent, J. L., Corbetta, M., Van Essen, D. C., and Raichle, M. E. (2005). The human brain is intrinsically organized into dynamic, anticorrelated functional networks. Proc. Natl. Acad. Sci. U.S.A. 102, 9673-9678. doi:10.1073/pnas.0504136102

Friston, K. J. (1995). Commentary and opinion. II. Statistical parametric mapping: ontology and current issues. J. Cereb. Blood Flow Metab. 15, 361-370. doi:10.1038/jcbfm.1995.45
Friston, K. J., Frith, C. D., Liddle, P. F., and Frackowiak, R. S. (1993). Functional connectivity: the principal-component analysis of large (PET) data sets. J. Cereb. Blood Flow Metab. 13, 5-14. doi:10.1038/jcbfm.1993.4

Gregoire, J., and van der Linden, M. (1997). Effect of age on forward and backward digit spans. Aging Neuropsychol. Cogn. 4, 140-149. doi:10.1080/ 13825589708256642

Greicius, M. D., Flores, B. H., Menon, V., Glover, G. H., Solvason, H. B., Kenna, H., et al. (2007). Resting-state functional connectivity in major depression: abnormally increased contributions from subgenual cingulate cortex and thalamus. Biol. Psychiatry 62, 429-437. doi:10.1016/j.biopsych.2006.09.020

Greicius, M. D., Krasnow, B., Reiss, A. L., and Menon, V. (2003). Functional connectivity in the resting brain: a network analysis of the default mode hypothesis. Proc. Natl. Acad. Sci. U.S.A. 100, 253-258. doi:10.1073/pnas. 0135058100

Gruart, A., Lopez-Ramos, J. C., Munoz, M. D., and Delgado-Garcia, J. M. (2008). Aged wild-type and APP, PS1, and APP + PS1 mice present similar deficits in associative learning and synaptic plasticity independent of amyloid load. Neurobiol. Dis. 30, 439-450. doi:10.1016/j.nbd.2008.03.001

Habas, C., Kamdar, N., Nguyen, D., Prater, K., Beckmann, C. F., Menon, V., et al. (2009). Distinct cerebellar contributions to intrinsic connectivity networks. J. Neurosci. 29, 8586-8594. doi:10.1523/JNEUROSCI.1868-09.2009

He, Y., Zang, Y., Liang, M., and Gong, G. (2004). Detecting functional connectivity of the cerebellum using low frequency fluctuations (LFFs). Lect. Notes Comput. Sci. 3217, 907-915. doi:10.1007/978-3-540-30136-3_110

Hedden, T., Van Dijk, K. R., Becker, J. A., Mehta, A., Sperling, R. A., Johnson, K. A., et al. (2009). Disruption of functional connectivity in clinically normal older adults harboring amyloid burden. J. Neurosci. 29, 12686-12694. doi:10.1523/JNEUROSCI.3189-09.2009

Helmstaedter, C. (2001). Verbaler Lern- und Merkfähigkeitstest: VLMT, Manual. Gottingen: Beltz Test GmbH.

Helmstaedter, C., and Durwen, H. F. (1990). VLMT: verbaler lern- und merkfähigkeitstest [VLMT: verbal learning and memory test]. Schweiz. Arch. Neurol. Psychiatr. 141, 21-30.

Hock, C., and Nitsch, R. M. (2000). Alzheimer dementia. Praxis (Bern 1994) 89, 529-540.

Jack, C. R. Jr., Lowe, V. J., Senjem, M. L., Weigand, S. D., Kemp, B. J., Shiung, M. M., et al. (2008). 11C PiB and structural MRI provide complementary information in imaging of Alzheimer's disease and amnestic mild cognitive impairment. Brain 131, 665-680. doi:10.1093/brain/awm336

Jagust, W. (2013). Vulnerable neural systems and the borderland of brain aging and neurodegeneration. Neuron 77, 219-234. doi:10.1016/j.neuron.2013.01.002

Jagust, W. J., Landau, S. M., Shaw, L. M., Trojanowski, J. Q., Koeppe, R. A., Reiman, E. M., et al. (2009). Relationships between biomarkers in aging and dementia. Neurology 73, 1193-1199. doi:10.1212/WNL.0b013e3181bc010c

Joel, S. E., Caffo, B. S., Van Zijl, P. C., and Pekar, J. J. (2011). On the relationship between seed-based and ICA-based measures of functional connectivity. Magn. Reson. Med. 66, 644-657. doi:10.1002/mrm.22818

Klunk, W. E., Engler, H., Nordberg, A., Wang, Y., Blomqvist, G., Holt, D. P., et al. (2004). Imaging brain amyloid in Alzheimer's disease with Pittsburgh compound-B. Ann. Neurol. 55, 306-319. doi:10.1002/ana.20009

Krstic, D., and Knuesel, I. (2013). Deciphering the mechanism underlying late-onsetalzheimer disease. Nat. Rev. Neurol. 9, 25-34. doi:10.1038/nrneurol. 2012.236

Lenglet, C., Abosch, A., Yacoub, E., De Martino, F., Sapiro, G., and Harel, N. (2012). Comprehensive in vivo mapping of the human basal ganglia and thalamic connectome in individuals using 7T MRI. PLoS ONE 7:e29153. doi:10.1371/journal.pone.0029153

Lezak, M. D. (1983). Neuropsychological Assessment, 2nd Edn. New York, NY: Oxford University Press.

MacKay, N. J. (2010). Scaling of human body mass with height: the body mass index revisited. J. Biomech. 43, 764-766. doi:10.1016/j.jbiomech.2009.10.038

Margulies, D. S., Kelly, A. M., Uddin, L. Q., Biswal, B. B., Castellanos, F. X., and Milham, M. P. (2007). Mapping the functional connectivity of anterior cingulate cortex. Neuroimage 37, 579-588. doi:10.1016/j.neuroimage.2007.05.019

Mathis, C. A., Wang, Y., Holt, D. P., Huang, G. F., Debnath, M. L., and Klunk, W. E. (2003). Synthesis and evaluation of 11C-labeled 6-substituted 2-aryl benzothiazoles as amyloid imaging agents. J. Med. Chem. 46, 2740-2754. doi:10.1021/jm030026b 
Matthews, P. M., Filippini, N., and Douaud, G. (2013). Brain structural and functional connectivity and the progression of neuropathology in Alzheimer's disease. J. Alzheimers Dis. 33(Suppl. 1), S163-S172. doi:10.3233/JAD-2012129012

Middleton, F. A., and Strick, P. L. (1994). Anatomical evidence for cerebellar and basal ganglia involvement in higher cognitive function. Science 266, 458-461. doi:10.1126/science.7939688

Mormino, E. C., Brandel, M. G., Madison, C. M., Rabinovici, G. D., Marks, S., Baker, S. L., et al. (2012). Not quite PIB-positive, not quite PIB-negative: slight PIB elevations in elderly normal control subjects are biologically relevant. Neuroimage 59, 1152-1160. doi:10.1016/j.neuroimage.2011.07.098

Mormino, E. C., Kluth, J. T., Madison, C. M., Rabinovici, G. D., Baker, S. L., Miller, B. L., et al. (2009). Episodic memory loss is related to hippocampalmediated beta-amyloid deposition in elderly subjects. Brain 132, 1310-1323. doi:10.1093/brain/awn320

Morris, J. C., Roe, C. M., Grant, E. A., Head, D., Storandt, M., Goate, A. M., et al. (2009). Pittsburgh compound B imaging and prediction of progression from cognitive normality to symptomatic Alzheimer disease. Arch. Neurol. 66, 1469-1475. doi:10.1001/archneurol.2009.269

Müller, H., Hasse-Sander, I., Horn, R., Helmstaedter, C., and Elger, C. E. (1997). Rey auditory-verbal learning test: structure of a modified German version. J. Clin. Psychol. 53, 663-671. doi:10.1002/(SICI)1097-4679(199711)53:7<663: :AID-JCLP4>3.0.CO;2-J

Nicholas, L. E., Brookshire, R. H., Schumacher, J. G., and Porrazzo, S. A. (1988). The Boston Naming Test: revised administration and scoring procedures and normative information for non-brain-damaged adults. Clin. Aphasiol. 18, 103-115.

Oh, H., Mormino, E. C., Madison, C., Hayenga, A., Smiljic, A., and Jagust, W. J. (2011). Beta-amyloid affects frontal and posterior brain networks in normal aging. Neuroimage 54, 1887-1895. doi:10.1016/j.neuroimage.2010.10.027

Petersen, R. C., Smith, G. E., Waring, S. C., Ivnik, R. J., Tangalos, E. G., and Kokmen, E. (1999). Mild cognitive impairment: clinical characterization and outcome. Arch. Neurol. 56, 303-308. doi:10.1001/archneur.56.3.303

Price, J. L., McKeel, D. W. Jr., Buckles, V. D., Roe, C. M., Xiong, C., Grundman, M., et al. (2009). Neuropathology of nondemented aging: presumptive evidence for preclinical Alzheimer disease. Neurobiol. Aging 30, 1026-1036. doi:10.1016/j.neurobiolaging.2009.04.002

Rabinovici, G. D., and Jagust, W. J. (2009). Amyloid imaging in aging and dementia: testing the amyloid hypothesis in vivo. Behav. Neurol. 21, 117-128. doi:10.3233/BEN-2009-0232

Roy, A. K., Shehzad, Z., Margulies, D. S., Kelly, A. M., Uddin, L. Q., Gotimer, K., et al. (2009). Functional connectivity of the human amygdala using resting state fMRI. Neuroimage 45, 614-626. doi:10.1016/j.neuroimage.2008.11.030

Scahill, R. I., Frost, C., Jenkins, R., Whitwell, J. L., Rossor, M. N., and Fox, N. C. (2003). A longitudinal study of brain volume changes in normal aging using serial registered magnetic resonance imaging. Arch. Neurol. 60, 989-994. doi:10.1001/archneur.60.7.989

Schmahmann, J. D. (1996). From movement to thought: anatomic substrates of the cerebellar contribution to cognitive processing. Hum. Brain Mapp. 4, 174-198. doi:10.1002/(SICI)1097-0193(1996)4:3

Schmahmann, J. D. (1998). Dysmetria of thought: clinical consequences of cerebellar dysfunction on cognition and affect. Trends Cogn. Sci. 2, 362-371. doi:10.1016/S1364-6613(98)01218-2

Schmahmann, J. D., and Caplan, D. (2006). Cognition, emotion and the cerebellum. Brain 129, 290-292. doi:10.1093/brain/awh729

Schmahmann, J. D., Doyon, J., McDonald, D., Holmes, C., Lavoie, K., Hurwitz, A. S., et al. (1999). Three-dimensional MRI atlas of the human cerebellum in proportional stereotaxic space. Neuroimage 10, 233-260. doi:10.1006/nimg.1999.0459

Schmahmann, J. D., MacMore, J., and Vangel, M. (2009). Cerebellar stroke without motor deficit: clinical evidence for motor and non-motor domains within the human cerebellum. Neuroscience 162, 852-861. doi:10.1016/j.neuroscience. 2009.06.023

Schmahmann, J. D., and Pandya, D. N. (2008). Disconnection syndromes of basal ganglia, thalamus, and cerebrocerebellar systems. Cortex 44, 1037-1066. doi:10.1016/j.cortex.2008.04.004

Schmahmann, J. D., Weilburg, J. B., and Sherman, J. C. (2007). The neuropsychiatry of the cerebellum - insights from the clinic. Cerebellum 6, 254-267. doi:10.1080/14734220701490995
Shaffer, J. L., Petrella, J. R., Sheldon, F. C., Choudhury, K. R., Calhoun, V. D., Coleman, R. E., et al. (2013). Predicting cognitive decline in subjects at risk for Alzheimer disease by using combined cerebrospinal fluid, MR imaging, and PET biomarkers. Radiology 266, 583-591. doi:10.1148/radiol.12120010

Sheline, Y. I., and Raichle, M. E. (2013). Resting state functional connectivity in preclinical Alzheimer's disease. Biol. Psychiatry 74, 340-347. doi:10.1016/j.biopsych. 2012.11.028

Sheline, Y. I., Raichle, M. E., Snyder, A. Z., Morris, J. C., Head, D., Wang, S., et al. (2010). Amyloid plaques disrupt resting state default mode network connectivity in cognitively normal elderly. Biol. Psychiatry 67, 584-587. doi:10.1016/j. biopsych.2009.08.024

Solbach, C., Uebele, M., Reischl, G., and Machulla, H. J. (2005). Efficient radiosynthesis of carbon-11 labelled uncharged thioflavin $\mathrm{T}$ derivatives using [11C]methyl triflate for beta-amyloid imaging in Alzheimer's disease with PET. Appl. Radiat. Isot. 62, 591-595. doi:10.1016/j.apradiso.2004.09.003

Sperling, R. A., Aisen, P. S., Beckett, L. A., Bennett, D. A., Craft, S., Fagan, A. M., et al. (2011). Toward defining the preclinical stages of Alzheimer's disease: recommendations from the national institute on aging-Alzheimer's association workgroups on diagnostic guidelines for Alzheimer's disease. Alzheimers Dement. 7, 280-292. doi:10.1016/j.jalz.2011.03.003

Sperling, R. A., Laviolette, P. S., O’Keefe, K., O’Brien, J., Rentz, D. M., Pihlajamaki, M., et al. (2009). Amyloid deposition is associated with impaired default network function in older persons without dementia. Neuron 63, 178-188. doi:10.1016/j.neuron.2009.07.003

Steffener, J., and Stern, Y. (2012). Exploring the neural basis of cognitive reserve in aging. Biochim. Biophys. Acta. 1822, 467-473. doi:10.1016/j.bbadis.2011.09.012

Stern, Y. (2009). Cognitive reserve. Neuropsychologia 47, 2015-2028. doi:10.1016/j. neuropsychologia.2009.03.004

Stevens, M. C., Pearlson, G. D., and Calhoun, V. D. (2009). Changes in the interaction of resting-state neural networks from adolescence to adulthood. Hum Brain Mapp. 30, 2356-2366. doi:10.1002/hbm.20673

Stoodley, C. J. (2012). The cerebellum and cognition: evidence from functional imaging studies. Cerebellum 11,352-365. doi:10.1007/s12311-011-0260-7

Stoodley, C. J., and Schmahmann, J. D. (2009). Functional topography in the human cerebellum: a meta-analysis of neuroimaging studies. Neuroimage 44, 489-501. doi:10.1016/j.neuroimage.2008.08.039

Stoodley, C. J., and Schmahmann, J. D. (2010). Evidence for topographic organization in the cerebellum of motor control versus cognitive and affective processing. Cortex 46, 831-844. doi:10.1016/j.cortex.2009.11.008

Stoodley, C. J., Valera, E. M., and Schmahmann, J. D. (2010). An fMRI study of intraindividual functional topography in the human cerebellum. Behav. Neurol. 23, 65-79. doi:10.3233/BEN-2010-0268

Stoodley, C. J., Valera, E. M., and Schmahmann, J. D. (2012). Functional topography of the cerebellum for motor and cognitive tasks: an fMRI study. Neuroimage 59, 1560-1570. doi:10.1016/j.neuroimage.2011.08.065

Storandt, M., Mintun, M. A., Head, D., and Morris, J. C. (2009). Cognitive decline and brain volume loss as signatures of cerebral amyloid-beta peptide deposition identified with Pittsburgh compound B: cognitive decline associated with Abeta deposition. Arch. Neurol. 66, 1476-1481. doi:10.1001/archneurol. 2009.272

Theysohn, N., Qin, S., Maderwald, S., Poser, B. A., Theysohn, J. M., Ladd, M. E., et al. (2013). Memory-related hippocampal activity can be measured robustly using FMRI at 7 Tesla. J. Neuroimaging 23, 445-451. doi:10.1111/jon.12036

Tombaugh, T. N. (2004). Trail Making Test A and B: normative data stratified by age and education. Arch. Clin. Neuropsychol. 19, 203-214. doi:10.1016/S08876177(03)00039-8

Tzourio-Mazoyer, N., Landeau, B., Papathanassiou, D., Crivello, F., Etard, O., Delcroix, N., et al. (2002). Automated anatomical labeling of activations in SPM using a macroscopic anatomical parcellation of the MNI MRI single-subject brain. Neuroimage 15, 273-289. doi:10.1006/nimg.2001.0978

Uddin, L. Q., Supekar, K., Amin, H., Rykhlevskaia, E., Nguyen, D. A., Greicius, M. D., et al. (2010). Dissociable connectivity within human angular gyrus and intraparietal sulcus: evidence from functional and structural connectivity. Cereb. Cortex 20, 2636-2646. doi:10.1093/cercor/bhq011

Unschuld, P. G., Buchholz, A. S., Varvaris, M., Van Zijl, P. C., Ross, C. A., Pekar, J. J., et al. (2013a). Prefrontal brain network connectivity indicates degree of both schizophrenia risk and cognitive dysfunction. Schizophr. Bull. doi:10.1093/ schbul/sbt077 
Unschuld, P. G., Liu, X., Shanahan, M., Margolis, R. L., Bassett, S. S., Brandt, J., et al. (2013b). Prefrontal executive function associated coupling relates to Huntington's disease stage. Cortex 49, 2661-2673. doi:10.1016/j.cortex.2013.05.015

van de Ven, V. G., Formisano, E., Prvulovic, D., Roeder, C. H., and Linden, D. E. (2004). Functional connectivity as revealed by spatial independent component analysis of fMRI measurements during rest. Hum. Brain Mapp. 22, 165-178. doi:10.1002/hbm.20022

Van Dijk, K. R., Hedden, T., Venkataraman, A., Evans, K. C., Lazar, S. W., and Buckner, R. L. (2010). Intrinsic functional connectivity as a tool for human connectomics: theory, properties, and optimization. J. Neurophysiol. 103, 297-321. doi:10.1152/jn.00783.2009

Vandenberghe, R., Van Laere, K., Ivanoiu, A., Salmon, E., Bastin, C., Triau, E., et al. (2010). 18F-flutemetamol amyloid imaging in Alzheimer disease and mild cognitive impairment: a phase 2 trial. Ann. Neurol. 68, 319-329. doi:10.1002/ana. 22068

Villemagne, V. L., Burnham, S., Bourgeat, P., Brown, B., Ellis, K. A., Salvado, O., et al. (2013). Amyloid beta deposition, neurodegeneration, and cognitive decline in sporadic Alzheimer's disease: a prospective cohort study. Lancet Neurol. 12, 357-367. doi:10.1016/S1474-4422(13)70044-9

Villemagne, V. L., Pike, K. E., Chetelat, G., Ellis, K. A., Mulligan, R. S., Bourgeat, P., et al. (2011). Longitudinal assessment of Abeta and cognition in aging and Alzheimer disease. Ann. Neurol. 69, 181-192. doi:10.1002/ana.22248

Whitfield-Gabrieli, S., and Ford, J. M. (2012). Default mode network activity and connectivity in psychopathology. Annu. Rev. Clin. Psychol. 8, 49-76. doi:10.1146/annurev-clinpsy-032511-143049

Whitfield-Gabrieli, S., and Nieto-Castanon, A. (2012). Conn: a functional connectivity toolbox for correlated and anticorrelated brain networks. Brain Connect. 2, 125-141. doi:10.1089/brain.2012.0073

Whitfield-Gabrieli, S., Thermenos, H. W., Milanovic, S., Tsuang, M. T., Faraone, S. V., McCarley, R. W., et al. (2009). Hyperactivity and hyperconnectivity of the default network in schizophrenia and in first-degree relatives of persons with schizophrenia. Proc. Natl. Acad. Sci. U.S.A. 106, 1279-1284. doi:10.1073/pnas. 0809141106

Winblad, B., Palmer, K., Kivipelto, M., Jelic, V., Fratiglioni, L., Wahlund, L. O., et al. (2004). Mild cognitive impairment - beyond controversies, towards a consensus: report of the International Working Group on Mild Cognitive Impairment. J. Intern. Med. 256, 240-246. doi:10.1111/j.1365-2796.2004.01380.x

Wirth, M., Oh, H., Mormino, E. C., Markley, C., Landau, S. M., and Jagust, W. J. (2013). The effect of amyloid beta on cognitive decline is modulated by neural integrity in cognitively normal elderly. Alzheimers Dement. 9, 687-698e681. doi:10.1016/j.jalz.2012.10.012

Woodward, T. S., Cairo, T. A., Ruff, C. C., Takane, Y., Hunter, M. A., and Ngan, E. T. (2006). Functional connectivity reveals load dependent neural systems underlying encoding and maintenance in verbal working memory. Neuroscience 139, 317-325. doi:10.1016/j.neuroscience.2005.05.043

World Medical Association. (1991). Declaration of Helsinki. Law Med. Health Care 19, 264-265.

Zhan, L., Mueller, B. A., Jahanshad, N., Jin, Y., Lenglet, C., Yacoub, E., et al. (2013). Magnetic resonance field strength effects on diffusion measures and brain connectivity networks. Brain Connect. 3, 72-86. doi:10.1089/brain.2012.0114

Conflict of Interest Statement: The authors declare that the research was conducted in the absence of any commercial or financial relationships that could be construed as a potential conflict of interest.

Received: 26 January 2014; paper pending published: 07 February 2014; accepted: 03 March 2014; published online: 18 March 2014.

Citation: Steininger SC, Liu X, Gietl A, Wyss M, Schreiner S, Gruber E, Treyer V, Kälin A, Leh S, Buck A, Nitsch RM, Prüssmann KP, Hock C and Unschuld PG (2014) Cortical amyloid beta in cognitively normal elderly adults is associated with decreased network efficiency within the cerebro-cerebellar system. Front. Aging Neurosci. 6:52. doi: 10.3389/fnagi.2014.00052

This article was submitted to the journal Frontiers in Aging Neuroscience.

Copyright (c) 2014 Steininger, Liu, Gietl, Wyss, Schreiner, Gruber, Treyer, Kälin, Leh, Buck, Nitsch, Prüssmann, Hock and Unschuld. This is an open-access article distributed under the terms of the Creative Commons Attribution License (CC BY). The use, distribution or reproduction in other forums is permitted, provided the original author(s) or licensor are credited and that the original publication in this journal is cited, in accordance with accepted academic practice. No use, distribution or reproduction is permitted which does not comply with these terms. 